\title{
Comportamento sexual e ocorrência de sífilis em estudantes universitários da área da saúde
}

\author{
Sexual behavior and occurence of syphilis in \\ students of university of health area
}

\author{
Thallys Denneyson Andrelino Silva' \\ Nadia Rodrigues Furtado Galeno ${ }^{2}$ (1) \\ Chrystiany Plácido de Brito Vieira ${ }^{3}$ (1) \\ Patrícia Maria Gomes de Carvalho ${ }^{4}$ (1) \\ Telma Maria Evangelista de Araujo 5 (1)
} ${ }^{1}$ Autor para correspondência. Universidade Federal do Piauí (Teresina).Piauí, Brasil. thallys-andrelino@hotmail.com
2-5Universidade Federal do Piauí (Teresina). Piauí, Brasil. nadiarfgaleno@hotmail.com, chrystianyplacido@yahoo.com,
patriciamariag80@hotmail.com, telmaevangelista@gmail.com

RESUMO | OBJETIVO: Analisar o comportamento sexual e a ocorrência de sífilis em estudantes da área da saúde de uma universidade pública do estado do Piauí. MÉTODO: Estudo epidemiológico, transversal, realizado no período de outubro de 2017 a fevereiro de 2018, com 357 estudantes universitários da área da saúde. Os dados foram coletados por meio de questionário, com perguntas fechadas e semiabertas, e teste rápido para Sífilis. Foram realizadas análises univariadas e bivariadas por meio da distribuição de frequências e teste exato de Fisher. Os dados foram analisados com o uso do Statistical Package for the Social Sciences. RESULTADOS: Verificou-se que $135(37,8 \%)$ estudantes afirmaram não utilizar o preservativo em todas as relações sexuais; 200 (56,0\%) foram classificados com comportamento sexual de risco; a frequência do uso de bebida alcoólica apresentou associação significativa com comportamento sexual de risco $(p=0,001)$. Foram detectados $2(0,6 \%)$ resultados reagentes para sífilis, ambos discentes do curso de Odontologia. CONCLUSÃO: Embora a ocorrência de sífilis nos estudantes tenha sido abaixo da esperada, a utilização de álcool previamente às relações sexuais pode comprometer práticas sexuais seguras.

DESCRITORES: Sífilis. Comportamento sexual. Fatores de risco. Consumo de álcool na faculdade. Estudantes de ciências da saúde.

\begin{abstract}
OBJECTIVE: To analyze the sexual behavior and the occurence of syphilis in students of health area at the brasilian public university. METHOD: Epidemiological, crosssectional study, held on the October 2017 to February 2018, with 357 university students of health area. The data were by questionary, with closed and semi open questions, and quick test for Syphilis. The data were analyzed with use of Statistical Package for Social Sciences. RESULTS: It was found $135(37,8 \%)$ students said don't use condoms in the all sexual realtions; $200(56 \%)$ were classified with sexual behavior of risc; The frequency of use of alcohol drink presented significant association with sexual behavior of risc $(p=0,001)$. Were delected $2(0,6 \%)$ results reagents for Syphilis, both students of dentistry course. CONCLUSION: Although the occurence of Syphilis in the students has been below expectations, the use of alcohol previously to sexual relations can compromise safe sexual practices.
\end{abstract}

DESCRIPTORS: Syphilis. Sexual behavior. Risk factors. Alcohol consumpition in college. Students of health area. 


\section{Introdução}

A sexualidade humana é um processo contínuo, influenciado por fatores biológicos, emocionais, ambientais, sociais e culturais que se refletem na vida e na saúde das pessoas. Constitui um tema de relevância social, especialmente na adolescência e na idade adulta, pela possibilidade de exposição e ocorrência de infecções sexualmente transmissíveis (IST) ${ }^{1}$.

Estima-se o surgimento de mais de um milhão de novos casos de IST por dia no planeta. Essas infecções possuem etiologias e apresentações clínicas variadas, e provocam um forte impacto na qualidade de vida, relações pessoais, familiares e sociais dos doentes ${ }^{2}$.

A ocorrência de IST é maior entre jovens de 14 a 29 anos em comparação às demais faixas etárias, pois foi observado que o início da atividade sexual e o número elevado de parcerias sexuais resulta em maior exposição aos agentes infecciosos nessa faixa etária. A população universitária constitui um grupo que apresenta alta exposição aos agentes dessas doenças, uma vez que o número elevado de parcerias sexuais está relacionado à maior vulnerabilidade ${ }^{3-4}$.

Estudos apontam que o ingresso em instituições de ensino superior pode contribuir para o aumento da vulnerabilidade de jovens às IST. Embora muitos estudantes universitários acreditem possuir o conhecimento necessário para a prevenção das IST, observa-se maior empenho relacionado aos cuidados para evitar gravidez indesejada, pela adesão ao anticoncepcional oral em detrimento do uso de preservativos ${ }^{3-5}$.

Se por um lado a vida universitária representa a oportunidade para se conquistar a independência familiar e financeira, observa-se por outro, que essa população está mais predisponente a uma busca por drogas psicoativas, dentre elas, as bebidas alcoólicas, com o objetivo de facilitar a aproximação entre as pessoas e como alternativa para aumentar a libido e o prazer. Ressalta-se que o consumo de álcool está associado diretamente ao aumento no número de parcerias sexuais e maior predisposição às IST $^{6}$.
Dentre as IST curáveis, está a sífilis, que é causada pelo Treponema pallidum e possui caráter sistêmico. É considerada um importante agravo em saúde pública no mundo e sua transmissão ocorre predominantemente por meio das vias sexual e vertical ${ }^{2}$. Estudo realizado com 640 universitários, entre 18 e 25 anos, observou que 22 (2,9\%) apresentaram sífilis e $116(17,6 \%)$ outras IST 1 .

No Brasil, foi observado nos últimos cinco anos um aumento constante no número de casos de sífilis adquirida. Entre os anos de 2015 e 2016, o crescimento do número absoluto de casos foi de $27,8 \%$. Somente no ano de 2016, foram notificados 87.593 casos. Além disso, as notificações de indivíduos nas faixas de 13 a 19 anos e 20 e 29 anos vêm apresentando tendência de aumento desde 2010. Entre 2010 e 2016, a elevação do percentual em cada uma dessas faixas etárias foi respectivamente de $39,9 \%$ e $13,8 \%{ }^{7}$.

Pesquisas de prevalência, conhecimentos e comportamentos sexuais relacionados à sífilis na população geral brasileira ainda são escassas e em população universitária são raras. Em diversas unidades federadas, inexistem estudos sobre a sífilis neste segmento populacional, e especialmente sobre a influência do comportamento sexual.

Diante do aumento da prevalência de sífilis no país e do quantitativo de jovens que apresentam comportamento sexual de risco, em função do uso de drogas lícitas e ilícitas, esta pesquisa teve como objetivo analisar o comportamento sexual e a ocorrência da sífilis em estudantes da área de saúde de uma universidade pública.

\section{Método}

\section{Delineamento do Estudo}

Trata-se de uma pesquisa epidemiológica do tipo transversal, realizada com o apoio do Programa Institucional de Bolsas de Iniciação Científica, e que se insere em um macroprojeto de pesquisa intitulado: "Análise da ocorrência e fatores relacionados ao HIV, Hepatite B, C e sífilis em estudantes de uma universidade pública". 


\section{Local do estudo}

A pesquisa foi desenvolvida no Centro de Ciências da Saúde de uma instituição pública de ensino superior do estado do Piauí, Brasil.

\section{População/Amostra do estudo}

A população do estudo foi composta pelo universo de estudantes dos cursos de bacharelado em Medicina, Farmácia, Enfermagem e Odontologia, matriculados a partir do $4^{\circ}$ semestre no Campus de Teresina, e que já haviam ingressado nas atividades práticas dos respectivos cursos, $(n=378)$. Contudo, após as perdas, relacionadas ao preenchimento incompleto do questionário, a população resultou em 53 estudantes de Medicina, 51 de Farmácia, 105 de Enfermagem e 148 de Odontologia, totalizando 357 participantes.

A opção por essa amostra se deu em virtude dessa pesquisa acompanhar o mesmo desenho do macroprojeto, considerando que os estudantes da área da saúde a partir do ingresso nos campos de prática já tiveram a oportunidade de construir conhecimentos sobre as IST, e também porque estão mais suscetíveis a acidentes biológicos com transmissão de algumas IST. Foram excluídos do estudo, alunos que efetuaram trancamento de curso no período da coleta de dados e aqueles com idade inferior a 18 anos.

\section{Coleta de dados}

A coleta dos dados ocorreu no período de outubro de 2017 a fevereiro de 2018, por meio de um questionário construído pelos autores, não validado, com questões fechadas e semiabertas, divido em oito partes e organizadas em diferentes eixos: Caracterização sócioeconômica e demográfica; dados relacionados ao uso de alcool e outras drogas; dados relacionados à exposição parenteral; dados relacionados ao comportamento sexual; dados relacionados a infecção sexualmente transmissível atual ou passada; dados relacionados ao conhecimento sobre infecção sexualmente transmissível; situação vacinal; marcadores sorológicos.

Associado ao questionário foi realizado a testagem rápida para sífilis, em data previamente agendada e local conveniente para os alunos. Inicialmente foram realizadas as devidas orientações sobre o questionário e testagem rápida para um grupo de estudantes, em seguida, os alunos eram conduzidos individualmente para uma sala privativa onde foi realizado o preenchimento do questionário, a testagem rápida para sífilis e a emissão do laudo do teste.

O processo para coleta de dados envolveu alunos de iniciação científica, mestrado e doutorado em Enfermagem, todos treinados para a aplicação do questionário e testagem rápida. Para a realização da testagem rápida para sífilis foram utilizados kits TR DPP $®$ - Bio- Manguinhos, fornecidos pela Secretaria de Estado da Saúde do Piauí.

Foram realizadas visitas prévias às coordenações dos cursos, para dar conhecimento sobre a pesquisa e para discutir os aspectos relacionados à logística da coleta e visitas às salas de aula, com o propósito de informar sobre a pesquisa, seus objetivos e solicitar a participação dos estudantes.

Considerando que o teste rápido para sífilis é de triagem, aqueles cujos resultados foram reagentes receberam orientação para procurar os serviços de atenção básica do município com vistas a realização de teste sorológico confirmatório e execução dos seguimentos necessários ${ }^{10}$.

\section{Variáveis do estudo}

A variável dependente foi o comportamento sexual, o qual foi classificado como seguro, nos casos em que os estudantes ainda não haviam iniciado a atividade sexual, ou que utilizam preservativo em todas as relações sexuais e que não praticam sexo sob efeito de álcool e/ou outras drogas. Aqueles que apresentaram uma ou mais práticas relacionadas ao uso inconsistente do preservativo, múltiplos parceiros e relações sexuais após consumo de álcool e/ou outras drogas foram classificados em comportamento sexual de risco ${ }^{8-9}$. As demais variáveis do estudo foram consideradas independentes.

\section{Organização e análise dos dados}

Os dados foram digitados com a utilização do Software Statistical Package for Social Science (SPSS) versão 22.0, em banco editado. Foram realizadas análises univariadas por meio da distribuição de frequências. $\mathrm{Na}$ análise bivariada utilizou-se o teste exato de Fisher para identificar associação entre o comportamento sexual seguro e de risco e o uso de álcool. A significância estatística foi fixada no nível de $p<0,05$. 


\section{Aspectos éticos e legais}

Para a realização do estudo, o macroprojeto teve a anuência da instituição de ensino superior onde os alunos são matriculados e foi aprovado pelo Comitê de Ética em Pesquisa da Universidade Federal do Piauí, sob o CAAE 47095915.8.0000.5214. A cada participante da pesquisa foi apresentado um Termo de Consentimento Livre e Esclarecido (TCLE), e garantida a confidencialidade, a privacidade, a proteção da imagem, a não estigmatização e a não utilização de informações em prejuízo das pessoas. Foram respeitados todos os aspectos éticos contidos na Resolução nº 466/12, do Conselho Nacional de Saúde ${ }^{11}$.

\section{Resultados}

Dentre os 357 estudantes que participaram do estudo, observou-se o predomínio de $198(55,5 \%)$ de estudantes na faixa etária entre 21 e 24 anos, 241 (67,5\%) sexo feminino, 204 (57,1\%) pardos, 340 (95,2\%) solteiros, 134 $(37,5 \%)$ com renda menor que três salários mínimos (Tabela 1).

Tabela 1. Caracterização socioeconômica e demográfica dos estudantes da área de saúde de uma instituição pública de ensino superior (n=357). Teresina, Piauí, Brasil, 2018

\begin{tabular}{|c|c|c|}
\hline Variáveis & $\mathbf{N}$ & $\%$ \\
\hline \multicolumn{3}{|l|}{ Idade } \\
\hline De 18 a 20 anos & 93 & 26,0 \\
\hline De 21 a 24 anos & 198 & 55.5 \\
\hline Mais de 24 anos & 66 & 18,5 \\
\hline \multicolumn{3}{|l|}{ Sexo } \\
\hline Masculino & 116 & 32,5 \\
\hline Feminino & 241 & 67,5 \\
\hline \multicolumn{3}{|l|}{ Cor/Etnia } \\
\hline Branco & 108 & 30,3 \\
\hline Negro & 43 & 12,0 \\
\hline Pardo & 204 & 57,1 \\
\hline Outra & 2 & 0,6 \\
\hline \multicolumn{3}{|l|}{ Estado Civil } \\
\hline Solteiro & 340 & 95,2 \\
\hline Casado/União estável & 16 & 4,5 \\
\hline $\begin{array}{c}\text { Separado } \\
\text { Renda Familiar }\end{array}$ & 1 & 0,3 \\
\hline$<3 \mathrm{SM}^{*}$ & 134 & 37,5 \\
\hline De 3 a 7 SM & 125 & 35,0 \\
\hline$>7 \mathrm{SM}$ & 56 & 15,7 \\
\hline Ignorado & 42 & 11,8 \\
\hline
\end{tabular}

Legenda: *Salários Mínimos. Fonte: Pesquisa Direta. 
Em relação ao comportamento sexual, verificou-se que $227(63,6 \%)$ estudantes afirmaram ter relações sexuais somente com pessoas do sexo oposto, $180(50,4 \%)$ tiveram sua primeira relação sexual na faixa etária de 16 a 19 anos, 193 (54,1\%) possuíam apenas uma parceria sexual, 81 (22,7\%) tiveram relações sexuais com parceiros eventuais nos últimos 12 meses. Quanto ao uso do preservativo, 135 (37,8\%) não utilizavam em todas as relações sexuais, $98(27,5 \%)$ relataram que o motivo para não utilizá-lo era a confiança no parceiro, $85(23,8 \%)$ referiram o consumo de bebida alcoólica antes das relações sexuais (Tabela 2).

Tabela 2. Comportamento sexual dos estudantes da área de saúde de uma instituição pública de ensino superior (n=357). Teresina, Piauí, Brasil, 2018

\begin{tabular}{|c|c|c|}
\hline Variáveis & $\mathbf{N}$ & $\%$ \\
\hline \multicolumn{3}{|l|}{ Você costuma ter relações sexuais } \\
\hline Somente com pessoas do mesmo sexo & 38 & 10,6 \\
\hline Somente com pessoas do sexo oposto & 227 & 63,6 \\
\hline Não importa o sexo & 11 & 3,1 \\
\hline Nem com homens nem com mulheres & 81 & 22,7 \\
\hline \multicolumn{3}{|l|}{ Idade quando teve a sua primeira relação sexual } \\
\hline 11 a 15 & 42 & 11,8 \\
\hline 16 a 19 & 180 & 50,4 \\
\hline 20 a 23 & 54 & 15,1 \\
\hline Não se aplica & 81 & 22,7 \\
\hline \multicolumn{3}{|l|}{ Tem apenas uma parceira sexual } \\
\hline $\operatorname{Sim}$ & 193 & 54,1 \\
\hline Não & 70 & 19,6 \\
\hline Não se aplica & 94 & 26,3 \\
\hline \multicolumn{3}{|c|}{ Transou nos últimos 12 meses com parceiros eventuais } \\
\hline $\operatorname{Sim}$ & 81 & 22,7 \\
\hline Não & 195 & 54,6 \\
\hline Não se aplica & 81 & 22,7 \\
\hline \multicolumn{3}{|l|}{ Usa preservativo em todas as relações sexuais } \\
\hline Sempre & 115 & 32,2 \\
\hline Nunca & 26 & 7,3 \\
\hline Às vezes & 135 & 37,8 \\
\hline Não se aplica & 81 & 22,7 \\
\hline \multicolumn{3}{|l|}{ Por que não usa preservativo* } \\
\hline Não gosta & 55 & 15,4 \\
\hline Confia no parceiro & 98 & 27,5 \\
\hline Transa com pessoas higiênicas & 8 & 2,2 \\
\hline Não se aplica & 196 & 54,9 \\
\hline \multicolumn{3}{|c|}{ Caso use bebida alcoólica, utiliza antes das relações sexuais } \\
\hline $\operatorname{Sim}$ & 85 & 23,8 \\
\hline Não & 173 & 48,5 \\
\hline Não se aplica & 99 & 27,7 \\
\hline
\end{tabular}

Legenda: *Dado direcionado às pessoas que responderam "nunca" ou "às vezes" na questão anterior. Fonte: Pesquisa Direta. 
Na tabela 3, observa-se que a maioria dos estudantes que já fez o uso de bebidas alcoolicas foi classificada com comportamento sexual de risco, totalizando $149(73,7 \%)$, e que a frequência com que se utiliza algum tipo de bebida alcoólica apresentou associação significativa com a classificação do comportamento sexual de risco $(p=0,001)$.

Tabela 3. Associação do comportamento sexual com o uso de álcool dos estudantes (n=357). Teresina, Piauí, Brasil, 2018

\begin{tabular}{|c|c|c|c|c|c|}
\hline \multirow[t]{2}{*}{ Comportamento sexual } & \multicolumn{2}{|c|}{ Seguro } & \multicolumn{2}{|c|}{ Risco } & \multirow[t]{2}{*}{ p-valor } \\
\hline & $\mathrm{n}$ & $\%$ & $\mathrm{n}$ & $\%$ & \\
\hline \multicolumn{6}{|l|}{ Fez ou faz uso de algum tipo de bebida alcoólica } \\
\hline Sim & 82 & 52,2 & 133 & 66,5 & 0,013 \\
\hline Não & 48 & 30,6 & 51 & 25,5 & \\
\hline Às vezes & 9 & 5,7 & 8 & 4,0 & \\
\hline Raramente & 18 & 11,5 & 8 & 4,0 & \\
\hline \multicolumn{6}{|l|}{ Frequência que utiliza bebida alcoólica, em média } \\
\hline Diariamente & 3 & 1,9 & 1 & 0,5 & 0,001 \\
\hline 3 a 5 vezes por semana & 38 & 24,5 & 58 & 28,7 & \\
\hline 1 a 2 vezes por semana & 33 & 21,3 & 32 & 15,9 & \\
\hline 1 vez por mês & 10 & 6,5 & 31 & 15,3 & \\
\hline Raramente & 23 & 14,8 & 29 & 14,4 & \\
\hline Não se aplica & 48 & 31,0 & 51 & 25,2 & \\
\hline
\end{tabular}

Legenda: b. Teste Exato de Fisher. Fonte: Pesquisa Direta.

De acordo com a tabela 4, foi observado que dentre os 357 estudantes que participaram do estudo, $53(14,8 \%)$ são graduandos de Medicina, 51 (14,3\%) de Farmácia, 105 (29,4\%) de Enfermagem e 148 (41,5\%) de Odontologia. A ocorrência de sífilis foi detectada em dois $(0,6 \%)$ estudantes, ambos discentes do curso de Odontologia, que dentre a população pesquisada, foi o curso que apresentou maior ocorrência de sífilis.

Tabela 4. Distribuição da ocorrência de sífilis segundo o curso dos estudantes (n=357).Teresina, Piauí, Brasil, 2018

\begin{tabular}{lcccccccc}
\hline \multirow{2}{*}{ Sífilis } & \multicolumn{10}{c}{ Medicina } & \multicolumn{2}{c}{ Farmácia } & \multicolumn{2}{c}{ Enfermagem } & \multicolumn{2}{c}{ Odontologia } \\
\cline { 2 - 9 } & $\mathrm{n}$ & $\%$ & $\mathrm{n}$ & $\%$ & $\mathrm{n}$ & $\%$ & $\mathrm{n}$ & $\%$ \\
\hline Reagente & - & - & - & - & - & - & 2 & 0,6 \\
Não Reagente & 53 & 14,8 & 51 & 14,3 & 105 & 29,5 & 146 & 40,9 \\
\hline Fonte: Pesquisa & & & & & & & &
\end{tabular}




\section{Discussão}

A maioria dos participantes encontrava-se na faixa etária de 21 a 24 anos, eram do sexo feminino e se autodeclararam pardos, o que está em conformidade com resultados encontrados em outras pesquisas realizadas com estudantes universitários no Brasil e em outros países ${ }^{9,12}$.

A maior parte dos estudantes declarou estar solteira e ter renda inferior a três salários mínimos. Esses fatores podem contribuir para a demonstração de um comportamento sexual de risco, pois pessoas solteiras possuem maior número de parcerias sexuais e a menor renda familiar está relacionada a uma maior pressão social para o uso de álcool e outras drogas ${ }^{13-14}$.

No que se refere ao comportamento sexual, a maioria dos participantes da pesquisa afirmou que praticava sexo apenas com pessoas do sexo oposto. Entretanto, uma parcela significativa informou que realizava práticas homossexuais e bissexuais. Estudos indicam que estudantes universitários homossexuais e bissexuais são mais vulneráveis a situações de risco ${ }^{9,15}$.

Grande parte dos estudantes afirmou ter iniciado atividades sexuais antes dos 18 anos, semelhante aos resultados encontrados em outros estudos com universitários ${ }^{4}$. A iniciação sexual precoce sem preservativo pode resultar em maiores chances para um comportamento sexual de risco, pois existe uma associação direta entre o uso do preservativo na primeira e na última relação sexual ${ }^{4-5}$.

A maioria dos estudantes declarou ter apenas uma parceria sexual, não utilizar o preservativo em todas as suas relações sexuais e que o motivo para o não uso do preservativo estava relacionado à confiança na sua parceria sexual. Este achado converge com outros estudos, cujos resultados indicam que muitos não utilizam preservativos por confiarem no parceiro ${ }^{5,16}$.

No entanto, estudo relata que muitos parceiros sexuais omitem informações sobre sua vida sexual pregressa e que existem altas taxas de sexo casual não assumido durante relacionamentos de compromisso, favorecendo o contágio das parcerias fixas e manutenção da cadeia de transmissão das IST. Desse modo, com o passar do tempo e aumento da confidencialidade pode ocorreruma sensaçãofalsa de segurança, que pode estimular as pessoas a manter relações desprotegidas ${ }^{16}$.
Todavia, o conhecimento adquirido por estudantes universitários é uma ferramenta importante para a adoção de comportamentos sexuais seguros, uso de preservativos e compreensão sobre as vias de transmissão das IST. Perceber e analisar os riscos envolvidos em comportamentos sexuais pode estimular os estudantes a adotar práticas sexuais mais seguras ${ }^{17-18}$.

Contudo, embora o conhecimento seja um importante instrumento na prevenção de IST entre os universitários, não é suficiente para reduzir a vulnerabilidade dessa população. Fato que corrobora tal afirmação foi o relato de alguns estudantes universitários da área da saúde que nunca utilizaram preservativos durante suas práticas sexuais ${ }^{19-20}$.

A universidade abre a possibilidade para adolescentes, jovens e adultos iniciarem uma formação profissional ao mesmo tempo que permite a descoberta de novas experiências. Muitos estudantes se mudam para outras cidades, passam a morar sozinhos ou com outros parentes e a adotar novos hábitos e rotinas para o seu cotidiano. Essa nova realidade pode influenciar o universitário a utilizar as drogas, dentre elas, as bebidas alcoólicas ${ }^{21}$.

O jovem ingresso no ambiente universitário frente a uma sensação de liberdade intensificada pelo afastamento da família e pela percepção de que a vida universitária constitui uma fase de autonomia, que pode propiciar a satisfação de suas curiosidades, sente-se estimulado a experimentar substâncias psicoativas para produzir diferentes sensações e possibilitar maior contato entre novas pessoas ${ }^{6}$.

O uso de bebidas alcoólicas e outras drogas antes das relações sexuais prejudica a utilização de preservativos, pois o consumo de substâncias psicoativas diminui a capacidade de julgamento racional e previsão de consequências de atos a serem realizados, o que propicia comportamentos sexuais de risco e o aumento das chances de exposição a agentes patológicos por meio do sexo ${ }^{8}$.

Estudos evidenciam que as IST são mais frequentes em estudantes que consumem bebidas alcoólicas de maneira abusiva. Também foi observado que a dependência relacionada a bebidas alcoólicas foi associada à maiores índices de relações sexuais desprotegidas e gravidez indesejada ${ }^{22-23}$. 
Destaca-se que a maioria dos estudantes universitários foram classificados com comportamento sexual de risco e que na amostra pesquisada foram detectados somente dois $(0,6 \%)$ casos reagentes para sífilis, o que se contrapôs a estudos populacionais, que apontam um aumento na ocorrência da infecção entre jovens no Brasil e no mundo ${ }^{2,7}$.

As limitações do estudo envolveram a baixa participação dos estudantes dos cursos de Medicina e Farmácia e preenchimento incompleto do formulário de pesquisa, resultando na ausência de respostas ou em afirmações que não condiziam com a realidade. Com vistas a minimizar este problema, os participantes receberam esclarecimentos prévios sobre a pesquisa e os questionários não foram identificados.

\section{Conclusão}

Os resultados desse estudo demonstram que é expressivo o contigente de estudantes universitários que possuem comportamento sexual de risco e que o uso de preservativos ocorre de maneira inconsistente, principalmente entre pessoas que possuem somente uma parceria sexual fixa, devido à confiança no relacionamento.

Foi observado que muitos estudantes consomem bebidas alcoólicas previamente às relações sexuais e que a frequência no consumo de bebidas alcoólicas associou-se estatisticamente com o comportamento sexual de risco. Apesar do baixo índice de reagentes para sífilis, esse dado é alarmante, pois revela que apesar do conhecimento adquirido em cursos de ensino superior da área da saúde, alguns estudantes mantêm comportamentos que sabidamente ocasionam maior exposição às IST.

É importante que as universidades e demais instituições de ensino percebam que devem ocorrer mudanças e adequações nas estratégias de educação em saúde que são realizadas para a população jovem e universitária. É necessário intervir de maneira mais efetiva para o aumento da frequência no uso do preservativo e a redução dos riscos relativos ao consumo de bebidas alcoólicas e outras drogas associadas às práticas sexuais.
Espera-se que as universidades e demais instituições de ensino propiciem um espaço para a divulgação de informações epidemiológicas e atividades de educação sexual contínua com o objetivo de estimular relações sexuais protegidas frente ao uso de preservativos, adoção de comportamentos sexuais mais seguros e estilos de vida mais saudáveis.

\section{Contribuições dos autores}

Silva TDA, Araujo TME e Galeno NRF participaram da concepção, delineamento, coleta de dados, busca e análise estatística dos dados da pesquisa, interpretação dos resultados e redação do artigo científico. Vieira CPB participou do delineamento, interpretação dos resultados e redação do artigo científico. Carvalho PMG participou da redação do artigo científico.

\section{Conflitos de interesses}

Nenhum conflito financeiro, legal ou político envolvendo terceiros (governo, empresas e fundações privadas, etc.) foi declarado para nenhum aspecto do trabalho submetido (incluindo, mas não se limitando a subvenções e financiamentos, participação em conselho consultivo, desenho de estudo, preparação de manuscrito, análise estatística, etc.).

\section{Referências}

1. Rutherford GW, Anglemyer A, Bagenda D, Muyonga M, Lindan $\mathrm{CP}$, Barker JL et al. University students and the risk of HIV and other sexually transmitted infections in Uganda: The Crane Survey. Int J Adolesc Med Health 2014;26(2):209-215. doi: 10.1515/ jjamh-2013-0515

2. Newman L, Rowley J, Hoorn SV, Wijesooriya NS, Unemo M, Low $\mathrm{N}$ et al. Global Estimates of the Prevalence and Incidence of Four Curable Sexually Transmitted Infections in 2012 Based on Systematic Review and Global Reporting. PLoS ONE. 2015;10(12): e0143304 doi: 10.1371/journal.pone.0143304

3. Reuter PR, McGinnis S, Reuter KE. Comparing the awareness of and beliefs in sexually transmitted infections among university students in Madagascar and the United States of America. Peer]. 2018;6:e4362. doi: $10.7717 /$ peerj.4362

4. Castro EL, Caldas TA, Morcillo AM, Pereira EMA, Velho PENF. $O$ conhecimento e o ensino sobre doenças sexualmente transmissíveis entre universitários. Ciênc Saúde Coletiva. 2016;21(6):1975-1984. doi: 10.1590/1413-81232015216.00492015 
5. Moreira LR, Dumith SC, Paludo SS. Uso de preservativos na última relação sexual entre universitários: quantos usam e quem são? Ciênc Saúde Coletiva. 2018;23(4):1255-1266. doi: $\underline{10.1590 / 1413-81232018234.16492016}$

6. Dázio EMR, Zago MMF, Fava SMCL. Uso de álcool e outras drogas entre universitários do sexo masculino e seus significados. Rev Esc Enferm USP. 2016;50(5):785-791. doi: 10.1590/S0080623420160000600011

7. Ministério da Saúde. Secretaria de Vigilância em Saúde. Departamento de Atenção Básica. Boletim Epidemiológico de Sífilis. [Internet]. 2017. [acesso em 2018 out 18]. Disponível: http:// portalarquivos.saude.gov.br/images/pdf/2017/novembro/13/BE2017-038-Boletim-Sifilis-11-2017-publicacao-.pdf

8. Dickson-Gomez J, McAuliffe T, Quinn K. The effects of housing status, stability and the social contexts of housing on drug and sexual risk behaviors. AIDS Behav. 2017;21(7):2079-2092. doi: 10.1007/s10461-017-1738-1

9. Sales WB, Caveião C, Visentin A, Mocelin D, Costa PM, Simm EB. Comportamento sexual de risco e conhecimento sobre IST/SIDA em universitários da saúde. Rev Enf Rev. 2016;IV(10):19-27. doi: 10.12707/RIV16019

10. Ministério da Saúde. Secretaria de Atenção à Saúde. Departamento de Atenção Básica.Orientações para a implantação dos testes rápidos de HIV e Sífilis na atenção básica: rede cegonha. [Internet]. 2013. [acesso em 2018 ago 16]. Disponível: http://189.28.128.100/dab/docs/portaldab/publicacoes/ orientacoes_implantacao_testes_rapido s_hiv_sifilis.pdf

11. Ministério da Saúde. Resolução $n^{\circ} 466$, de 12 de dezembro de 2012. Aprova as diretrizes e normas regulamentadoras de pesquisas envolvendo seres humanos. [Internet] Brasília: Ministério da Saúde. 2012. [acesso em 2018 nov 01]. Disponível: http://conselho.saude.gov.br/resolucoes/2012/reso466.pdf

12. Shiferaw Y, Alemu A, Assefa A, Tesfaye B, Gibermedhin E, Amare M. Perception of risk of HIV and sexual risk behaviors among University students: implication for planninginterventions. BMC Res Notes. 2014;7:162. doi: 10.1186/1756-0500-7-162

13. Penha JC, Aquino CBQ, Neri EAR, Reis TGO, Aquino PC, Pinheiro AKB. Fatores de risco para doenças sexualmente transmissíveis em profissionais do sexo do interior piauiense. Rev Gaúcha Enferm. 2015;36(2):63-9. doi: 10.1590/1983$\underline{1447.2015 .02 .52089}$

14. Arroyave LJO, Restrepo-Méndez MC, Horta BL, Menezes AMB, Gigante DP, Gonçalves H. Tendências e desigualdades nos comportamentos de risco em adolescentes: comparação das coortes de nascimentos de Pelotas, Rio Grande do Sul, Brasil. Cad Saúde Pública. 2016;32(9):e00120215. doi: 10.1590/0102311X00120215
15. Assis SG, Gomes R, Pires TO. Adolescência, comportamento sexual e fatores de risco à saúde. Rev Saúde Pública. 2014;48(1):43-51. doi: 10.1590/S0034-8910.2014048004638

16. Santos MJO, Ferreira EMS, Ferreira MMC. Comportamentos contraceptivos de estudantes portugueses do ensino superior. Rev Bras Enferm. 2018;71(supl 4):1805-13. doi: 10.1590/0034$\underline{7167-2017-0623}$

17. Wu X, Hong F, Lan L, Zhang C, Feng T, Yang Y. Poor awareness of syphilis prevention and treatment knowledge among six diferente populations in south China. BMC Public Health. 2016;16:287. doi: 10.1186/s12889-016-2966-4

18. Yang Z, Huang Z, Dong Z, Zhang S, Han J, Jin M. Prevalence of high-risky behaviors in transmission of HIV among high school and college student MSM in China: a meta-analysis. BMC Public Health. 2015;15:1272. doi: 10.1186/s12889-015-2614-4

19. Kuete M, Huang Q, Rashid A, Ma XL, Yuan H, Antezana JPE. Differences in Knowledge, Attitude, and Behavior towards HIV/ AIDS and Sexually Transmitted Infections between Sexually Active Foreign and Chinese Medical Students. BioMed Research International. 2016;2016:4524862. doi: 10.1155/2016/4524862

20. Santos VP, Coelho MTAD, Macário EL, Oliveira TCS. Existe relação entre o conhecimento de estudantes a respeito das formas de contágio do HIV/AIDS e suas respostas sobre a proximidade com soropositivos? Ciênc Saúde Coletiva. 2017;22(8):2745-2752. doi: 10.1590/1413-81232017228.25892015

21. Zeferino MT, Hamilton $H$, Brands B, Wright MGM, Cumsille $F$, Khenti $\mathrm{A}$. Consumo de drogas entre estudantes universitários: família, espiritualidade e entretenimento moderando a Influência dos pares. Texto Contexto - Enferm. 2015;24(Esp):125-35. doi: 10.1590/0104-07072015001150014

22. Castaño-Perez GA, Calderon-Vallejo GA. Problemas associados ao consumo de álcool em estudantes universitários. Rev Latino-Am Enfermagem. 2014;22(5):739-46. doi: 10.1590/01041169.3579 .2475

23. Sullivan C, Martin N, White C, Newbury-Birch D. Assessing the delivery of alcohol screening and brief intervention in sexual health clinics in the north east of England. BMC Public Health. 2017;17:884. doi: 10.1186/s12889-017-4878-3 